\title{
Circulating zinc-a2-glycoprotein levels are low in newly diagnosed patients with metabolic syndrome and correlate with adiponectin
}

\author{
$\mathrm{Lu} \mathrm{Lei}^{1 \dagger}$, Kejia Li ${ }^{2 \dagger}$, Ling Li ${ }^{3+}$, Xia Fang ${ }^{2}$, Tingting Zhou ${ }^{2}$, Cheng Zhang ${ }^{4}$, Yong Luo ${ }^{4}$, Hua Liu ${ }^{5}$, Xiaoqiang Li ${ }^{6}$, \\ Hongting Zheng ${ }^{7}$, Lin Zhang ${ }^{8}$, Gangyi Yang ${ }^{1,2^{*}}$ and Lin Gao ${ }^{1 *}$
}

\begin{abstract}
Background: Zinc-a2-glycoprotein (ZAG) is a novel adipokine that reduces insulin resistance, protecting against type 2 diabetes. However, past studies have been contradictory. This cross-sectional study aims to investigate the association of circulating ZAG with metabolic syndrome (MetS) in middle-aged and older Chinese adults.

Methods: Four hundred eighty nine individuals (234 healthy controls and 255 MetS patients) were examined. All individuals were screened for MetS according to the diagnostic guidelines of the United States National Cholesterol Education Program (NCEP) Expert Panel Adult Treatment Panel (ATP) III criteria. Circulating ZAG and ADI levels were measured by ELISA. Blood fat, glucose and insulin were measured with a commercial kit. Circulating ZAG levels were compared with various parameters in study subjects.

Results: Plasma ZAG levels were lower in MetS patients compared to those of the healthy controls (35.0 \pm 11.7 vs. $46.1 \pm 18.6 \mathrm{mg} / \mathrm{L}, P<0.01)$. ZAG showed a positive correlation with age, HDL-C, HOMA- $\beta$ and ADI, but a negative correlation with Fat\%, BMI, WHR, blood pressure, triglycerides, FFA, FBG, 2 h-BG, fasting insulin, 2 h-Ins, $\mathrm{HbA1c}$ and HOMA-IR $(P<0.01)$. When the population was divided according to tertiles of ADI, subjects in the highest tertile had the highest ZAG levels. The analysis of ROC curves revealed that the best cutoff value for plasma ZAG to predict MetS was $45.2 \mathrm{mg} / \mathrm{L}$ (sensitivity $92 \%$, specificity $59 \%$, and AUC 0.80).

Conclusions: We found that circulating ZAG levels were decreased in patients with MetS. In fact, circulating ZAG decreased progressively with an increasing number of MetS components and associated with ADI levels, suggesting that ZAG is related to IR and MetS and may be a sensitizer.
\end{abstract}

Trial Registration: ChiCTR-OCC-11001422. Registered 23 June 2011.

Keywords: Zinc-a2-glycoprotein, Adiponectin, Metabolic syndrome, Insulin resistance

\section{Background}

Metabolic syndrome (MetS) is a clustering of cardiovascular risk factors including visceral obesity, disturbances of carbohydrate and lipid metabolism, and hypertension. The cardiovascular risk is proportional to the number of MetS components [1]. People diagnosed with MetS are known

\footnotetext{
*Correspondence: gangyiyang@163.com; gaolinpro@yeah.net

${ }^{\dagger}$ Equal contributors

'Department of Endocrinology, the Affiliated Hospital, Zunyi Medical College, Guizhou, China

Full list of author information is available at the end of the article
}

to be more susceptible to developing type 2 diabetes mellitus (T2DM) and face a 2-fold increased risk for cardiovascular disease (CVD) $[2,3]$. MetS patients are also more susceptible to developing hypertension and cancers [4-6]. A meta-analysis has shown that the rate of cardiovascular disease, coronary heart disease (CHD) and stroke in MetS subjects is $50 \%$ higher than in healthy controls [4]. Although extensive research efforts have been put forth to elucidate the pathogenesis of MetS, it is still largely unknown. In recent years, some adipocyte-related cytokines have been shown to be biomarkers relative to MetS such 
as adiponectin (ADI). Insulin resistance (IR), the consequence of visceral obesity, has been known as a major pathomechanism in the development of MetS components [7]. The changes of circulating levels in some cytokines may indicate a potential role for MetS pathogenesis.

Zinc- $\alpha 2$-glycoprotein (ZAG) is a $40-\mathrm{kD}$ soluble glycoprotein isolated from human plasma [8] and has also been found in other secretions, such as saliva, milk, sweat, urine, and cerebrospinal fluids [9]. One study showed that ZAG is also an adipokine because it is secreted by animal adipose tissues [10]. Although the biological functions of ZAG are largely unknown, it has been shown that ZAG is a novel adipokine and that its expression in adipose tissue is down-regulated in obese mice [10]. In addition, it has also been demonstrated that ZAG knockout mice are susceptible to weight gain when fed a high fat diet (HFD) which is associated with decreased lipolysis [11]. Importantly, Balazet et al. reported that silencing ZAG led to a reduction of ADI, insulin receptor substrate-1(IRS-1) and glucose transporters-4 (GLUT4) gene/protein expression in primary human adipocytes indicating that ZAG plays a pivotal role in regulating adipose tissue and whole-body insulin sensitivity [12].

In a human study, it has been demonstrated that ZAG is negatively relative to body weight and induces lipolysis in obese patients [13]. Recently, we have reported that circulating ZAG levels are markedly lower in patients with newly diagnosed type 2 diabetes mellitus (nT2DM) than in healthy controls. Circulating ZAG positively correlated with plasma ADI levels and negatively correlated with obesity related parameters and homeostasis model assessment of insulin resistance (HOMA-IR) [14]. ADI is an adipocyte-secreted hormone that has important associations with obesity and insulin resistance. A strong positive correlation between circulating ZAG and ADI suggests that ZAG may also be an insulin sensitizer. More recently, we have shown that circulating ZAG levels were much lower in women with polycystic ovary syndrome (PCOS) and IR than in healthy women and positively correlated with $\mathrm{M}$-value, determined by an euglycemic-hyperinsulinemic clamp (EHC) considered the 'gold standard' measure of insulin sensitivity [15]. Therefore, ZAG has been considered to be a new candidate for involvement in the pathogenesis of IR and dysmetabolism. However, previous studies in humans and rodents have produced conflicting results regarding the link between ZAG and IR, T2DM, and obesity [9-15]. Currently, only one study has addressed the association between circulating ZAG and MetS [16]. As with most new discoveries, this finding needs to be reproduced and further investigated. The aim of the current study is to investigate the association between the number and type of MetS components and circulating ZAG levels in an elderly population. In addition, the relationship between circulating ZAG levels and ADI is also investigated in MetS patients.

\section{Methods \\ Participants}

Four hundred eighty nine individuals, 255 subjects with MetS (aged 39-82, male/female 125/130) and 234 healthy controls (aged 37-80, male/female 124/110), were recruited for this study from outpatients attending the Internal Medicine Department or routine medical checkup at the Second Affiliated Hospital, Chongqing Medical University, or from the community or schools through advertisement during February 2014 to December 2015. The diagnosis of MetS was based on the United States National Cholesterol Education Program (NCEP) Expert Panel Adult Treatment Panel (ATP) III criteria [17]. MetS individuals have more than three of following characteristics: 1) central obesity (waist circumference exceeds 90 or $80 \mathrm{~cm}$ for Asian male and female, respectively); 2) hypertension(systolic pressure equals or exceed $130 \mathrm{mmHg}$ or diastolic pressure equals or exceeds $85 \mathrm{mmHg}$ );3) elevated blood glucose (fasting glucose level equals or exceeds5.5 mmol/L [100 mg/dL]), or T2DM; 4) elevated plasma triglycerides (TG; level equals or exceeds $1.69 \mathrm{mmol} / \mathrm{L}$ [150 mg/dL]) and 5) low level of highdensity lipoprotein-cholesterol (HDL-C level equals or is less than $1.04 \mathrm{mmol} / \mathrm{L}[40 \mathrm{mg} / \mathrm{dL}]$ for maleand $1.29 \mathrm{mmol} /$ $\mathrm{L}[50 \mathrm{mg} / \mathrm{dL}]$ for female) are regarded as MetS positive. The exclusion criteria included cancer, current renal or liver disease, and a history of myocardial infarction, stroke, or transient ischemic attacks, or a history of regular use of medications for diabetes, hypertension, and/or lipidlowering drugs. The patients with MetS were newly diagnosed and had not been treated with any agents. Agematched healthy subjects without clinical evidence of major diseases were used as the controls.

Subjects were classified according to body mass index (BMI) (lean <25; and overweight/obese $\geq 25 \mathrm{~kg} / \mathrm{m}^{2}$ ). Subjects were also divided into subgroup according to gender (male: $n=149$; female: $n=240$ ). All subjects gave their written informed consent before entering the study. This study was conducted in accordance with the Declaration of Helsinki and approved by the human research ethics committee of Chongqing Medical University.

\section{Anthropometric and biochemical measurements}

Anthropometric measurements including height and body weight were taken. The BMI, waist circumference and waist-to-hip ratio (WHR) were calculated. HOMA-IR was calculated using the following eq. [18]: HOMA-IR = fasting insulin (FIns, mU/L) $\times$ fasting blood glucose (FBG, mmol/ $\mathrm{L}) / 22.5$. The homeostasis model assessment of insulin secretion (HOMA- $\beta$ ) were calculated using the following 
equation: HOMA- $\beta=[20 \times$ FIns $(\mu \mathrm{U} / \mathrm{ml})] /[\mathrm{FBG}(\mathrm{mmol} / \mathrm{L})$ - 3.5]. Plasma was stored at $-80{ }^{\circ} \mathrm{C}$ for the determination of free fatty acid (FFA), insulin, ZAG, ADI and blood fat levels. Plasma glucose and glycosylated haemoglobin (HbA1c) were immediately measured by the glucoseoxidase method and anion-exchange HPLC, respectively. Plasma insulin levels were measured using chemiluminescence. Free fatty acids (FFAs) were measured with a commercial kit (Randox Laboratories Ltd., Antrim, UK). Total cholesterol, low-density lipoprotein cholesterol (LDL-C), TG, and HDL-C were determined enzymatically using an auto-analyzer (Hitachi 747; Hitachi, Tokyo, Japan).

\section{Measurements of plasma adipokines}

Circulating ZAG concentration was measured with an ELISA obtained from Ray Biotech Inc. following the manufacturer's protocol. The limit of detection was $0.02 \mathrm{mg} /$ $\mathrm{mL}$, and intra- assay and inter-assay variations were $2.56 \%$ and $6.63 \%$ respectively. Plasma ADI levels were also measured with an ELISA from Adipobiotech [19].

\section{Statistical analysis}

All analyses were performed with SPSS version 15.0 (SPSS, Chicago, IL). Data are expressed as mean \pm SD or median (interquartile range). Normal distribution of the data was tested using Kolmogorox-Smirnov test. Several variables were skewed and logarithmically transformed to obtain a normal distribution. Comparisons between groups were performed with Non-parametric tests or Student $t$ test. Correlations between variables were assessed using Pearson correlation analyses by controlling for the covariates. Multiple linear regression was performed to determine variables that had independent associations with circulating ZAG, and included were all variables with significant associations or correlations with circulating ZAG and those with possible biological relevance. Multiple stepwise regression analysis was performed with ageadjusted plasma ZAG levels as the dependent variables, and by entering the independent variable with the highest partial correlation coefficient at each step, with a F-value probability for inclusion of 0.05 and 0.01 for removal. The trend of circulating ZAG levels associated with MetS was analyzed using the Row Mean Scores and CochranArmitage trend test. The ORs for ZAG levels and MetS were calculated by binary logistic regression. Receiver operator characteristic (ROC) curve analysis was employed to identify the optimal cut-off values of ZAG to diagnose MetS. $P<0.05$ was considered significant.

\section{Results}

\section{Characteristics and circulating ZAG levels of study participants}

Anthropometric and metabolic parameters of the study subjects were showed in Table 1 . There were no significant
Table 1 Main clinical features and circulating ZAG levels in MetS and control subjects

\begin{tabular}{|c|c|c|c|}
\hline Variable & Controls $(n=234)$ & Mets $(n=255)$ & $P$-value \\
\hline male/female & $124 / 110$ & $125 / 130$ & NS \\
\hline Age (year) & $51.9 \pm 9.2$ & $52.4 \pm 7.4$ & NS \\
\hline BMI (kg/m2) & $23.6 \pm 3.5$ & $26.1 \pm 3.7$ & $<0.001$ \\
\hline FAT (\%) & $29.2 \pm 6.9$ & $30.6 \pm 5.1$ & 0.014 \\
\hline WHR & $0.88 \pm 0.06$ & $0.91 \pm 0.06$ & $<0.001$ \\
\hline SBP $(m m H g)$ & $119.6 \pm 12.8$ & $127.6 \pm 13.2$ & $<0.001$ \\
\hline $\mathrm{DBP}(\mathrm{mmHg})$ & $75.2 \pm 7.5$ & $78.0 \pm 8.2$ & $<0.001$ \\
\hline TC $(\mathrm{mmol} / \mathrm{L})$ & $4.88 \pm 0.92$ & $5.00 \pm 0.63$ & NS \\
\hline TG $(\mathrm{mmol} / \mathrm{L})$ & $1.38 \pm 0.62$ & $2.07 \pm 0.54$ & $<0.001$ \\
\hline $\mathrm{HDL}-\mathrm{C}(\mathrm{mmol} / \mathrm{L})$ & $1.47 \pm 0.34$ & $1.32 \pm 0.27$ & $<0.001$ \\
\hline LDL-C (mmol/L) & $2.74 \pm 0.76$ & $2.72 \pm 0.69$ & NS \\
\hline FFA $(\mu \mathrm{mol} / L)$ & $0.54 \pm 0.27$ & $0.67 \pm 0.29$ & $<0.001$ \\
\hline FBG (mmol/L) & $6.15(5.30-8.57)$ & 8.98 (8.12-9.89) & $<0.001$ \\
\hline $2 \mathrm{~h}-\mathrm{BG}(\mathrm{mmol} / \mathrm{L})$ & $9.52(6.14-14.86)$ & $16.86(12.76-20.44)$ & $<0.001$ \\
\hline FIns (mU/L) & $10.90(7.00-13.86)$ & $13.11(9.76-16.47)$ & $<0.001$ \\
\hline 2 h-Ins (mU/L) & $40.89(34.8-51.71)$ & $45.73(37.85-55.89)$ & 0.011 \\
\hline HbA1c (\%) & $6.98 \pm 1.52$ & $7.84 \pm 1.21$ & $<0.001$ \\
\hline HOMA-IR & $3.08(1.80-4.93)$ & $5.45(3.68-7.05)$ & $<0.001$ \\
\hline HOMA- $\beta$ & 60.76 (47.51-99.76) & $50.44(37.89-59.90)$ & $<0.001$ \\
\hline Adiponectin $(\mu \mathrm{g} / \mathrm{L})$ & $34.9 \pm 11.4$ & $31.1 \pm 9.5$ & $<0.001$ \\
\hline ZAG (mg/L) & $46.1 \pm 18.6$ & $35.0 \pm 11.8$ & $<0.001$ \\
\hline
\end{tabular}

MetS metabolic syndrome, BMI Body mass index, FAT\% the percentage of fat in vivo, WHR Waist hip ratio, SBP Systolic blood pressure, DBP Diastolic blood pressure, TC Total cholesterol, TG Triglyeride, HDL-C High-density lipoprotein cholesterol, LDL-C Low-density lipoprotein cholesterol, FFA free fatty acid, FBG fasting blood glucose, $2 h-B G$ 2-h blood glucose after glucose overload, Fins fasting insulin, $2 \mathrm{~h}$-Ins 2-h plasma insulin after glucose overload, HbAlc glycosylated haemoglobin, HOMA-IR homeostasis model assessment of insulin resistance, HOMA- $\beta$ HOMA $\beta$ cell insulin secretion index

differences in age, TC and LDL-C levels between control and MetS groups. However, as expected, BMI, the percentage of fat in vivo (FAT\%), WHR, systolic blood pressure (SBP), diastolic blood pressure (DBP), TG, FFA, FBG, $2 \mathrm{~h}$ blood glucose after a $75 \mathrm{~g}$ glucose load (2 h-BG), FIns, 2-h plasma insulin after glucose overload (2-h Ins), HbA1c and HOMA-IR were significantly increased, whereas HDL-C and HOMA- $\beta$ were significantly decreased in MetS subjects when compared with the controls $(P<0.05$ or $P<0.01)$. Importantly, MetS patients had lower circulating ZAG and ADI levels than the control subjects (both $P<0.01$; Fig. 1a and b). Furthermore, circulating ZAG levels were significantly lower in MetS with overweight/ obese $(n=156)$ and control subjects with overweight/obese ( $n=79)$ than their lean subjects (MetS, $n=99$; control, $n=155)($ MetS: $31.4 \pm 10.1$ vs. $40.8 \pm 11.9 \mu \mathrm{g} / \mathrm{L}$; Controls: $35.1 \pm 12.0$ vs. $51.6 \pm 18.9$, both $P<0.01$ ) (Fig. 1c). Similar changes were found in circulating ADIlevels (MetS: $28.3 \pm 8.1 \mathrm{vs} .35 .5 \pm 10.0 \mu \mathrm{g} / \mathrm{L}$; Controls: $28.94 \pm 6.82$ vs. 

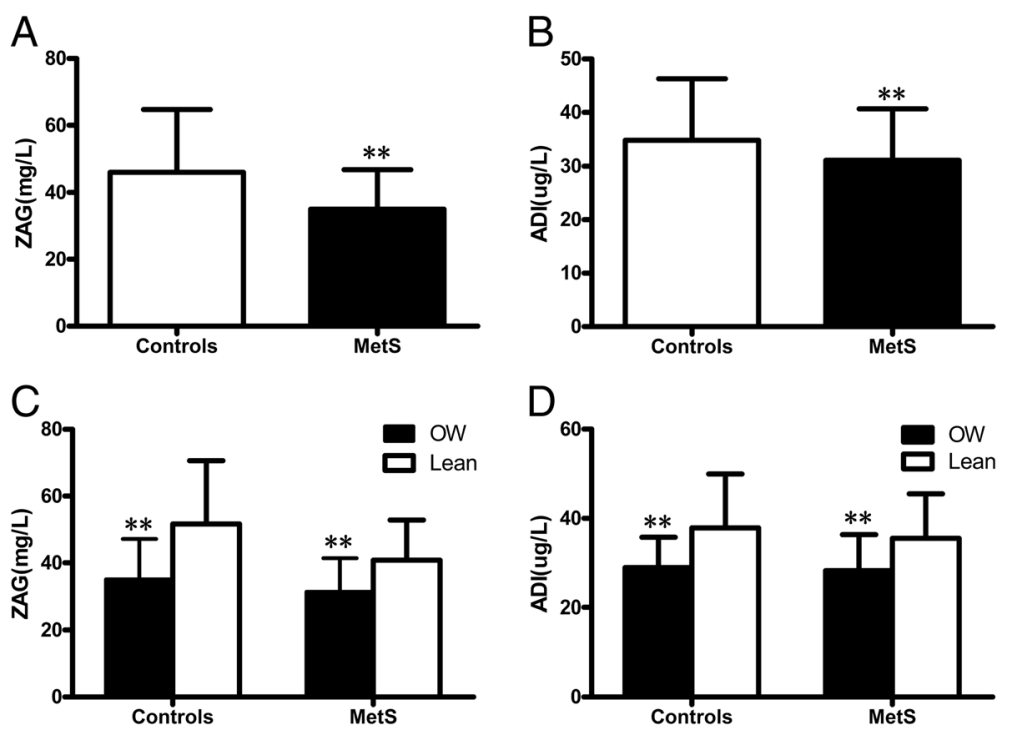

Fig. 1 a Circulating ZAG levels in MetS patients and controls; b Circulating ADI levels in MetS patients and controls (vs. controls: ${ }^{*}, P<0.01$ ); c Circulating ZAG levels in MetS patients and controls according to BMl; $\mathbf{d}$ Circulating ADI levels in MetS patients and controls according to BMI (lean: $\mathrm{BMI}<25 \mathrm{~kg} / \mathrm{m}^{2}$ and overweight/obese: BMI $\geq 25 \mathrm{~kg} / \mathrm{m}^{2}$; vs. lean: ${ }^{* *}, P<0.01$ ). Overweight/obese: $n=156$ for MetS; $n=79$ for the controls; lean: $n=99$ for MetS; $n=155$ for the controls

$37.9 \pm 12.1$, both $P<0.01$ ) (Fig. 1d). Circulating ZAG levels were similar in both men and women $(39.02 \pm 16.48$ vs. $41.76 \pm 16.18 \mu \mathrm{g} / \mathrm{L})$, whereas circulating ADI levels were higher in women than in men $(36.06 \pm 11.36 \mathrm{vs}$. $30.04 \pm 9.05 \mu \mathrm{g} / \mathrm{L}, P<0.01)$.

\section{Correlation of ZAG with clinical parameters}

Next, we investigated the association of plasma ZAG levels with various anthropometric and biochemical parameters by using partial correlations. Plasma ZAG correlated negatively with markers of adiposity (WHR, BMI, FAT\% and FFA, all $P<0.01$ ) and glucose metabolic parameters (FBG, 2 h-BG, HbA1c, FIns and HOMA- $\beta$, all $P<0.01$; Table 2). It also closely correlated with insulin resistance indices (decreased HOMA-IR) and blood pressure (all $P<0.01$; Table 2). In addition, Circulating ZAG levels correlated positively with age, HOMA- $\beta$ and HDL-C (all $P<0.01$; Table 2). Importantly, circulating ZAG also correlated positively with plasma ADI levels, an insulin sensitizer and cardio-protective adipokine $(P<0.01$; Table 2$)$. However, there was no significant correlation between ZAG levels and TC, LDL-C and $2 \mathrm{~h}$-Ins.In multiple stepwise regression analysis, only BMI, TC, HOMA-IR and ADI were independent related factors with circulating ZAG levels (Table 2). The multiple regression equation was: $\mathrm{Y}_{\mathrm{ZAG}}=4.012-0.037 \times \mathrm{BMI}+0.060 \times \mathrm{TC}$ $-0.046 \times$ HOMA-IR $+0.013 \times$ ADI $(R=0.750$, $\left.R^{2}=0.563\right)$.
Table 2 Linear regression analysis of variables associated with circulating ZAG levels in the study population

\begin{tabular}{|c|c|c|c|c|}
\hline \multirow[t]{2}{*}{ Variable } & \multicolumn{2}{|l|}{ Simple } & \multicolumn{2}{|c|}{ Multiple } \\
\hline & $r$ & $P$-value & $\beta$ & $P$-value \\
\hline Age (year) & 0.229 & $<0.001$ & & \\
\hline BMI $\left(\mathrm{kg} / \mathrm{m}^{2}\right)$ & -0.565 & $<0.001$ & -0.037 & $<0.001$ \\
\hline FAT (\%) & -0.213 & $<0.001$ & & \\
\hline WHR & -0.221 & $<0.001$ & & \\
\hline SBP $(m m H g)$ & -0.237 & $<0.001$ & & \\
\hline $\mathrm{DBP}(\mathrm{mmHg})$ & -0.094 & 0.039 & & \\
\hline TC (mmol/L) & 0.054 & NS & 0.060 & $<0.001$ \\
\hline TG $(\mathrm{mmol} / \mathrm{L})$ & -0.215 & $<0.001$ & & \\
\hline $\mathrm{HDL}-\mathrm{C}(\mathrm{mmol} / \mathrm{L})$ & 0.130 & 0.004 & & \\
\hline LDL-C (mmol/L) & 0.005 & NS & & \\
\hline FFA (umol/L) & -0.137 & 0.002 & & \\
\hline $\mathrm{FBG}(\mathrm{mmol} / \mathrm{L})$ & -0.498 & $<0.001$ & & \\
\hline $2 \mathrm{~h}-\mathrm{BG}(\mathrm{mmol} / \mathrm{L})$ & -0.475 & $<0.001$ & & \\
\hline FIns (mU/L) & -0.413 & $<0.001$ & & \\
\hline $2 \mathrm{~h}-\operatorname{Ins}(\mathrm{mU} / \mathrm{L})$ & -0.047 & NS & & \\
\hline HbA1c (\%) & -0.574 & $<0.001$ & & \\
\hline HOMA-IR & -0.538 & $<0.001$ & -0.046 & $<0.001$ \\
\hline HOMA- $\beta$ & 0.211 & $<0.001$ & & \\
\hline Adiponectin ( $\mu \mathrm{g} / \mathrm{L})$ & 0.610 & $<0.001$ & 0.013 & $<0.001$ \\
\hline
\end{tabular}

In multiple linear regression analysis, values included for analysis were age, sex, BMI, WHR, BP, FBG, insulin, HOMA-IR, HOMA- $\beta$, FFA, total cholesterol, HDL-C, LDL-C, triglyceride and Adiponectin 
ZAG characteristics according to plasma ADI quartiles and MetS components

In order to understand the association between ZAG and ADI, the population was divided according to tertiles of ADI (T1: 0-26.52; T2, 26.53-31.75; T3, >31.75 $\mu \mathrm{g} / \mathrm{L}$ ). Age adjusted estimates of ZAG means showed significant association between levels of ZAG and ADI among all subgroups. Overall, subjects in the highest tertiles of ADI had the highest ZAG levels (Fig. 2c). To further explore the association of circulating ZAG levels with MetS, we stratified the mean levels of circulating ZAG by the number of components of the MetS. The result indicated that circulating ZAG reduced progressively with increasing number of the MetS components ( $P$ for trend <0.01) (Fig. 2a). Patientswith $0,1,2$, 3or more component (s) of the MetS had reduced circulating ZAG levels of $58.3 \pm 15.9$; $49.2 \pm 20.1 ; 41.5 \pm 16.7 ; 36.4 \pm 13.3 ; 33.1 \pm 8.8 \mu \mathrm{g} / \mathrm{L}$ (mean $\pm \mathrm{SD}$ ), respectively (Fig. 2a).

\section{Binary logistic regression and ROC curves analysis}

Binary logistic regression analysis showed that plasma ZAG concentrations were significantly associated with MetS even after controlling for anthropometric variables, lipidprofile and hormone levels (Table 3). When considering MetS patients and controls as a whole, regression analyses, including all-factor and stepwise models, showed that the main predictors of circulating ZAG levels were HOMA-IR, ADI, HbA1c, TC and MetS (Fig. 2b). Furthermore, when concentrations were analyzed both by a Row Mean Scores test and a Cochran-Armitage trend test, the reduced levels of circulating ZAG showed a significant linear trend and were independently associated with MetS (Table 4). To explore the predictive value of ZAG for MetS, we analyzed the receiver operating characteristic (ROC) curves of plasma ZAG. The results revealed that the best cutoff value for circulating ZAG to predict MetS was $45.2 \mathrm{mg} / \mathrm{L}$ (sensitivity $92 \%$, specificity $59 \%$, and AUC 0.80; Fig. 2d).

\section{Discussion}

One study has revealed that MetS patients have higher serum ZAG levels than individuals without MetS and circulating levels of ZAG elevated progressively with an increasing number of MetS components [17]. However, as with most new discoveries, these findings need to be reproduced and confirmed. Therefore, we consider it important to examine a large population to determine whether circulating ZAG correlates with MetS, lipid profiles and ADI (another adipocyte-secreted hormone that has important associations with MetS). In the

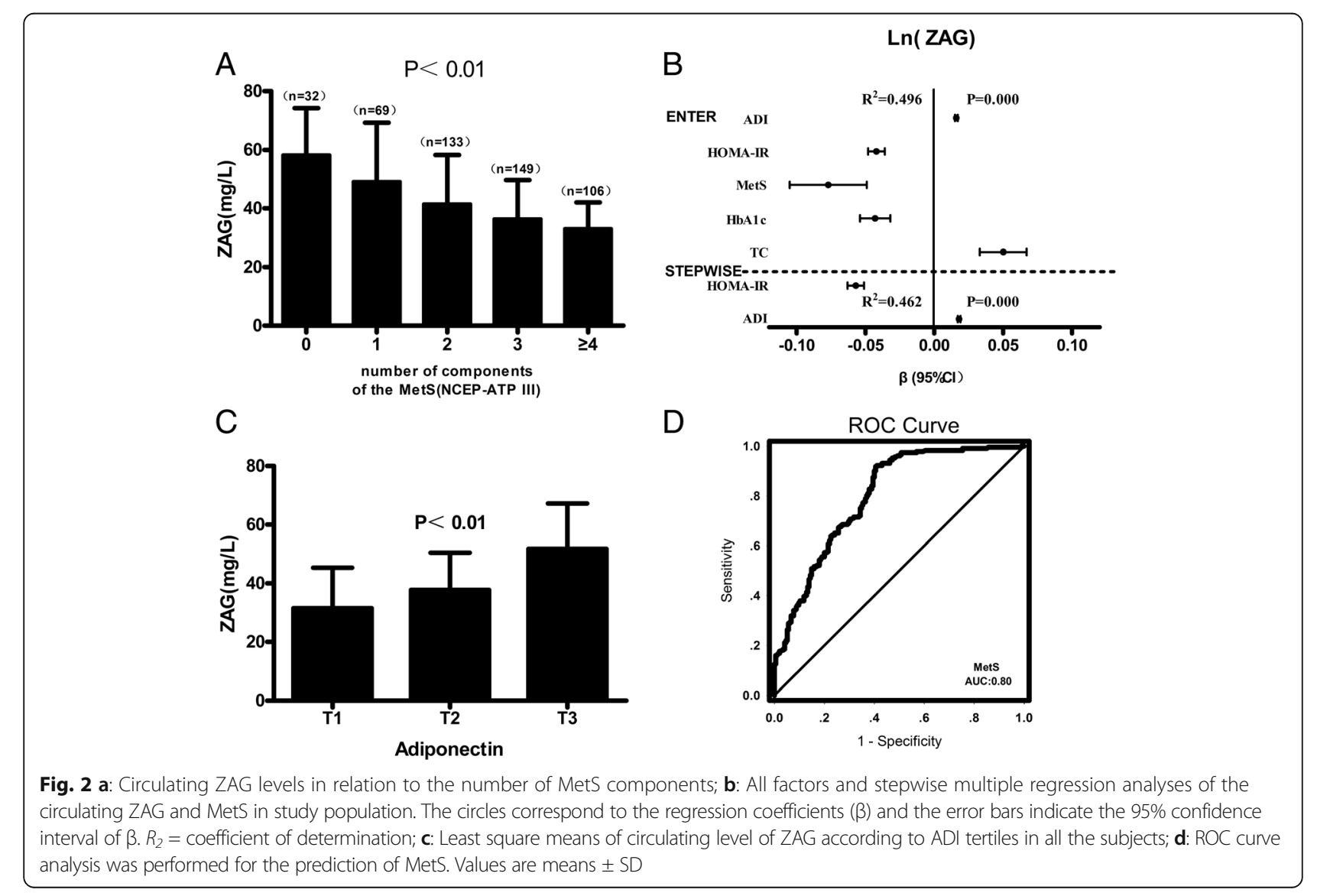


Table 3 Association of circulating ZAG with MetSin fully adjusted models

\begin{tabular}{llll}
\hline Model adjust & \multicolumn{2}{l}{ Mets } & \\
\cline { 2 - 4 } & OR & $95 \% \mathrm{Cl}$ & $P$-value \\
\hline Age, SBP, DBP & 0.953 & $0.939-0.968$ & $<0.001$ \\
Age, SBP, DBP, WHR, BMI & 0.970 & $0.953-0.987$ & 0.001 \\
Age, SBP, DBP, WHR, BMI, lipid profile & 0.982 & $0.962-1.004$ & 0.103
\end{tabular}

Results of multivariate logistic regression analysis are presented as the odds ratio (OR) of being in MetS status decrease in circulating. $B M I$ body mass index, WHR waist-to-hip, SBP systolic blood pressure, DBP diastolic blood pressure; lipid profile, including total cholesterol, triglyceride, LDL- and HDL-cholesterol

current study, we found that circulating ZAG levels were significantly lower in subjects with MetS than in those without MetS or in subjects with a higher number of MetS components such as IR and central obesity. We speculate that 1) the reduction in plasma ZAG concentration in MetS patients might be due to a defensive response, which may represent an ability to adapt to metabolic disturbance; 2) decreasing circulating ZAG might also suggest an increasing consumption of antagonizing metabolic stress, such as hyperglycemia and hyperlipidemia; 3) dysmetabolism might lead to the dysregulation of ZAG in biosynthesis and release. These factors need to be further clarified with in vivo and vitro studies. However, to our surprise, these results are not in agreement with previous findings in which circulating ZAG was found to be elevated in patients with MetS [17]. The differences in study design, including in patients election (e.g., aging, obesity or lean, glycaemia and lipidemia levels, sample size, agent treatment, other diseases, and complications) and experimental conditions likely contribute to the disparity. Furthermore, derangements in glucose homeostasis or analtered lipid profile may exert influence on the release of ZAG from body tissues. In the current study, MetS patients were newly diagnosed and were not treated with oral agents or special diets. These factors may in fact strengthen the analysis in our study. However, the reduced circulating ZAG levels in MetS subjects need to be further investigated.

The present study data also indicates that circulating ZAG correlates positively with age, HDL-C, and HOMA- $\beta$ and negatively with parameters of adiposity (WHR, FAT\% and BMI), IR (increased fasting insulin

Table 4 Row mean scores and Cochran-Armitage trend testof the impact of plasma ZAG level on MetS

\begin{tabular}{lll}
\hline & Mets & \\
\cline { 2 - 3 } & $X^{2}$ & $P$-value \\
\hline ROW Mean Scores Test & 56.4118 & $<0.001$ \\
Cochran-Armitage Test & -32.5801 & $<0.001$ \\
\hline
\end{tabular}

The circulating ZAG levels of all subjects were cut-offand adjusted for age, sex, BMI, WHR, SBP, DBP, TC, TG,LDL-C and HDL-C and HOMA-IR), dyslipidemia (increased TG and FFA), DBP, and SBP. Lower ZAG levels in MetS patients were associated with parameters of glucose metabolism (increasing FBG, $2 \mathrm{~h}-\mathrm{BG}$ and $\mathrm{HbA1c}$ ). In addition, it has been reported that ADI is a well-established marker for IR. Therefore, it is important to verify the association between ZAG and ADI. In the current study, we found that circulating ZAG levels correlated positively with plasma ADI levels in our study population, further confirming that ZAG is related to IR and may be a sensitizer. These results also suggest that circulating ZAG levels reflect the changes of MetS components and support the hypothesis that ZAG should be considered a marker of IR directly involved in the homeostasis of glucose and lipid metabolism.

To determine the predictive value of ZAG for MetS, we analyzed the ROC curves of circulating ZAG and MetS. The results showed that ZAG may be a good predictor for the diagnosis of MetS. The optimal cutoff value of ZAG for identifying individuals with MetS was also determined. Our study also has some limitations. First, a cross-sectional design limits any firm conclusion about the possible causative role of ZAG in MetS. Longitudinal intervention studies and future investigation are necessary. Secondly, our sample consisted entirely of Chinese people. Therefore, the extrapolation of these results to other ethnic groups should be undertaken with caution. Third, ZAG was not a prespecified end point of recruited MetS subjects in the study, and measurements of ZAG were made on stored samples, although the samples were relatively fresh. Furthermore, the current analyses are based on single measurements of circulating ZAG, which may not reflect ZAG levels over time. Serial changes in circulation need to be measured at different stages of MetS. Nevertheless, the strengths of this study include 1) newly diagnosed MetS subjects prevent pharmacotherapy and other confounding variables or complications; 2) the association between circulating ZAG and MetS is investigated; 3) the predictive value of circulating ZAG for MetS is evaluated; 4) relatively large sample size.

\section{Conclusion}

In summary, our data showed that circulating ZAG levels are decreased in patients with MetS. Circulating ZAG decreased progressively with an increasing number of MetS components and it was associated with ADI levels, suggesting that ZAG is related to IR and MetS and may be an insulin sensitizer. Therefore, ZAG may be a useful marker for the prediction of MetS.

\section{Abbreviations}

$2 \mathrm{~h}$-BG: 2-h blood glucose after glucose overload; $2 \mathrm{~h}$-Ins: 2-h plasma insulin after glucose overload; ADI: Adiponectin; BMl: Body mass index; Fat\%: The percentage of fat in vivo; FBG: Fasting blood glucose; FFA: Free fatty acid; 
HDL-C: High-density lipoprotein cholesterol; HOMA-IR: Homeostasis model assessment of insulin resistance; HOMA- $\beta$ : HOMA $\beta$ cell insulin secretion index; ROC: Receiver operating characteristic; WHR: Waist hip ratio

\section{Acknowledgements}

Not applicable.

\section{Funding}

This work was supported by research grants from the National Natural Science Foundation of China 81570752, the Grants from the Natural Science Foundation Project of CQ (No. cstc2015jcyjA10084) and the Science and Technology Key Program of Health Bureau of Chongqing(2015ZDXM038).

\section{Availability of data and materials}

All dataset are presented in the main paper.

\section{Authors' contributions}

$L L, K L, X F, C Z, X L, T Z, L Z$, and $Y L$ performed experiments, analyzed data and participated in text. $L L, H Z$ and $X Z$ participated in manuscript writing and revision. GY and LG conceived the project, supervised the overall project and wrote the manuscript. All authors read and approved the final manuscript.

\section{Ethics approval and consent to participate}

All procedures performed in studies involving human participants were in accordance with the ethical standards of the institutional.

\section{Consent for publication}

All the subjects have signed informed consent and all the authors of the article have consent to publish the manuscript

\section{Competing interests}

The authors declare that they have no competing interests.

\section{Publisher's Note}

Springer Nature remains neutral with regard to jurisdictional claims in published maps and institutional affiliations.

\section{Author details}

'Department of Endocrinology, the Affiliated Hospital, Zunyi Medical College, Guizhou, China. ${ }^{2}$ Department of Endocrinology, the Second Affiliated Hospital, Chongqing Medical University, Chongqing, China. ${ }^{3}$ Key Laboratory of Diagnostic Medicine (Ministry of Education) and Department of Clinical Biochemistry, College of Laboratory Medicine, Chongqing Medical University, Chongqing, China. ${ }^{4}$ The Center of Clinical Research of Endocrinology and Metabolic Diseases in Chongqing and Department of Endocrinology, Chongqing three Gorges Central Hospital, Chongqing, China. ${ }^{5}$ Department of Pediatrics, University of Mississippi Medical Center, 2500 North State Street, Jackson, Mississippi MS 39216-4505, USA. ${ }^{6}$ Children's Hospital of Chongqing Medical University, Chongqing, China. ${ }^{7}$ Department of Endocrinology, Xingiao Hospital, Third Military Medical University, Chongqing, China. ${ }^{8}$ Department of Endocrinology, the Ba Yan NaoEr Hospital, Inner Mongolia 015000, China.

\section{Received: 9 January 2017 Accepted: 3 August 2017}

\section{Published online: 14 August 2017}

\section{References}

1. Grundy SM. Hypertriglyceridemia, insulin resistance, and the metabolic syndrome. Am J Cardiol. 1999;83(9B):25F-9F.

2. Alberti KG, Zimmet PZ. Definition, diagnosis and classification of diabetes mellitus and its complications. Part 1: diagnosis and classification of diabetes mellitus provisional report of a WHO consultation. Diab Med J Br Diab Assoc. 1998;15(7):539-53.

3. Tan A, Gao Y, Yang X, Zhang H, Qin X, Mo L, et al. Low serum osteocalcin level is a potential marker for metabolic syndrome: results from a Chinese male population survey. Metabolism. 2011;60(8):1186-92.

4. Alberti KG, Zimmet P, Shaw J, Group IDFETFC. The metabolic syndrome-a new worldwide definition. Lancet. 2005;366(9491):1059-62.

5. Siu PM, Yu AP, Benzie IF, Woo J. Effects of 1-year yoga on cardiovascularrisk factors in middle-aged and older adults with metabolic syndrome: a randomized trial. DiabetolMetabSynd. 2015;30(7):40.
6. Expert Panel on Detection, Evaluation, and Treatment of High Blood Cholesterol in Adults. Executive summary of the third report of the National Cholesterol Education Program (NCEP) expert panel on detection, evaluation, and treatment of high blood cholesterol in adults (adult treatment panel III). JAMA. 2001;285(19):2486-97.

7. Reaven GM. Role of insulin resistance in human disease. Diabetes. 1988; 37(12):1595-607.

8. Burgi W, Schmid K. Preparation and properties of Zn-a2-glycoprotein of normal human plasma. J Biol Chem. 1961;236:1066-74.

9. Tada T, Ohkubo I, Niwa M, Sasaki M, Tateyama H, Eimoto T. Immunohistochemical localization of Zn-a2-glycoprotein in normal human tissues. J Histochem Cytochem. 1991;39(9):1221-6.

10. Bing C, Bao Y, Jenkins J, Sanders P, Manieri M, Cinti S, et al. Zinc- a2glycoprotein, a lipid mobilizing factor, is expressed in adipocytes and is upregulated in mice with cachexia. Proc Natl Acad Sci U S A. 2004;101(8):2500-5.

11. Rolli V, Radosavljevic M, Astier V, Macquin C, Castan-Laurell W, et al. Lipolysis is altered in MHC class I zinc-alpha(2)-glycoprotein deficient mice. FEBS Lett. 2007;581(3):394-400

12. Balaz M, Vician M, Janakova Z, Kurdiova T, Surova M, Imrich R, et al. Subcutaneous adipose tissue zinc-a 2-glycoprotein is associated with adipose tissue and whole-body insulin sensitivity. Obesity (Silver Spring). 2014:22(8):1821-9.

13. Selva DM, Lecube A, Hernández C, Baena JA, Fort JM, Simó R. Lower zincalpha2 -glycoprotein production by adipose tissue and liver in obese patients unrelated to insulin resistance. J Clin Endocrinol Metab. 2009;94(11): 4499-507.

14. Yang M, Liu R, Li S, Luo Y, Zhang Y, Zhang L, et al. Zinc-a2-glycoprotein is associated with insulin resistance in humans and is regulated by hyperglycemia, hyperinsulinemia or liraglutide administration: crosssectional and interventional studies in normal, insulin-resistant, and newly diagnosed diabetic subjects. Diabetes Care. 2013;36(5):1074-82.

15. Lai Y, Chen J, Li L, Yin J, He J, Yang M, et al. Circulating zinc-A2-glycoprotein levels and insulin resistance in polycystic ovary syndrome. Sci Rep. 2016; 16(6):25934.

16. Yeung DC, Lam KS, Wang Y, Tso AW, Xu A. Serum zinc-alpha2-glycoprotein correlates with adiposity, triglycerides, and the key components of the metabolic syndrome inChinese subjects. J Clin Endocrinol Metab. 2009;94(7): 2531-6.

17. Grundy SM, Cleeman JI, Daniels SR, Donato KA, Eckel RH, Franklin BA, et al. Diagnosis and management of the metabolic syndrome: an American Heart Association/National Heart, Lung, and Blood Institute Scientific Statement. Circulation. 2005;112:2735-52.

18. Albareda M, Rodríguez-Espinosa J, Murugo M, de Leiva A, Corcoy R. Assessment of insulin sensitivity and $\beta$-cell function from measurements in the fasting state and during an oral glucose tolerance test. Diabetologia. 2000;43:1507-11.

19. Tso AW, Sham PC, Wat NM, Xu A, Cheung BM, Rong R, et al. Polymorphisms of the gene encoding adiponectin and glycaemic outcome of Chinese subjects with impaired glucose tolerance: a 5-year followup study. Diabetologia. 2006:49:1806-15.

\section{Submit your next manuscript to BioMed Central and we will help you at every step:}

- We accept pre-submission inquiries

- Our selector tool helps you to find the most relevant journal

- We provide round the clock customer support

- Convenient online submission

- Thorough peer review

- Inclusion in PubMed and all major indexing services

- Maximum visibility for your research

Submit your manuscript at www.biomedcentral.com/submit
C Biomed Central 